\title{
The difficulties associated with integrating computation into undergraduate physics.
}

\author{
Ashleigh Leary, ${ }^{1}$ Paul W. Irving, ${ }^{1}$ and Marcos D. Caballero ${ }^{1,2,3}$ \\ ${ }^{1}$ Department of Physics and Astronomy, Michigan State University, East Lansing, MI 48824 \\ ${ }^{2}$ CREATE for STEM Institute, Michigan State University, East Lansing, MI 48824 \\ ${ }^{3}$ Department of Physics \& Center for Computing in Science Education, University of Oslo, N-0316 Oslo, Norway
}

From a department being resistant to change to students not buying into computational activities, the challenges that are faced with integrating computation into the undergraduate physics curriculum are varied. The Partnership for Integration of Computation into Undergraduate Physics (PICUP) aims to expand the role of computation in the undergraduate physics curriculum. The research presented in this paper is part of a larger project examining the role of the PICUP workshop in facilitating both the integration of computation into classrooms and developing a supportive community to support this integration. An important part of providing the necessary supports for integration is understanding and categorizing the problems members of this community of integrators face when integrating computation in their courses. Through individual and group interviews, we discuss the barriers to integration that new and experienced community members of PICUP have experienced in the past or perceive could exist in the future.

\section{INTRODUCTION}

The integration of computation into the undergraduate physics curriculum is becoming an important priority for the community of undergraduate physics educators [1]. There is a large pool of people who teach undergraduate physics who are shifting toward utilizing computation in some sense in their physics classes [2]. This shift is being motivated in some part by the NGSS standards, which are promoting this idea of helping the next generation of scientists develop computational thinking [3-5]. Those standards, designed with K-12 education in mind, are influencing instructors at the college level as well [6]. However, as with any change in teaching practice, there are difficulties and challenges to be negotiated in order to integrate successfully.

The need for facilitation of this negotiation sparked the formation of The Partnership for Integration of Computation into Undergraduate Physics (PICUP). The purpose of PICUP is to create a dialogue for those interested in integrating computation, those who are in the midst of integrating, and those who have already integrated successfully. This dialogue consists of pedagogy, methodology, and issues that are important when it comes to integrating computation into the undergraduate physics curricula. The PICUP group also uses this dialogue to accomplish their overall goal of getting undergraduates comfortable and competent with computation. PICUP realizes that computation is the third arm of physics, alongside theory and experiment. They also realize that students who are not learning this skill are at a disadvantage in this increasingly data-rich and model-driven society.

The PICUP Faculty Development Workshop (FDW) is a week-long event that is focused on introducing faculty to curriculum and pedagogical ideas in the hope that the attendees will then successfully implement those ideas when they return to their home institutions [7]. The workshop provides an opportunity for attendees to engage with implementers who hold expertise and experience in implementing computation into their curriculum. During the workshop, there is no explicit discussion of the challenges and difficulties one may face when integrating computation but the PICUP community places an emphasis on providing post-workshop support. But despite these efforts, the members of the PICUP community still lack knowledge around integrating computation smoothly and effectively.

\section{LITERATURE REVIEW}

There has been prior research about curriculum reform when integrating or reforming teaching methods using approaches based on Physics Education Research (PER) [8]. The work completed by Dancy and Henderson on the constraints of implementing research-informed practices plays an important role in examining the challenges of teaching physics in general [9]. They highlight the fact that PER is a thriving research field that produces a number of results, indicating the effectiveness of PER-informed approaches, but the results obtained are not widely adopted.

Dancy and Henderson examined this conundrum by interviewing faculty members and asking them about their opinions regarding PER based approaches [8]. Their research identified that the main issue lies with inconsistency between the beliefs of the instructors and the actual enacted practice of those beliefs. The reasons for these inconsistencies fell into five main systemic forces: student resistance, time structure, departmental norms, expectations of content coverage, and lack of instructor time. The fact that they are called "systemic forces" is telling. Dancy and Henderson make sure to acknowledge that these instructors do not act in isolation rather, they are in the system of their institution. That system can choose to support or resist PER-informed practices, and the unfortunate reality is that the systems that instructors find themselves in typically resist.

In the following paragraphs the five systemic forces from Dancy and Henderson [9] are discussed in order to help answer the two questions this paper intends to address: how do the existing barriers found by Dancy and Henderson apply to integrating computation in the physics curriculum, and what new barriers appear when trying to integrate computation into 
the physics curriculum.

Student Resistance: Students resist by not supporting research-based methods. Dancy and Henderson specify this by stating that there's a norm that has been built for these students. They do not have to be willing participants and instead can expect to go to class and not engage with activities if they choose not to. PER-based approaches often encourage making the classroom more interactive for them and initial resistances by the students is common place.

Time Structure: This refers to the fact that it is not possible to change how many weeks there will be of class. Semester structures at institutions are not flexible and are of a fixed length of time, which does not allow for individual differences in learning needs. Combine this with students who are taking multiple other courses and that limits the amount of time they can spend on one class.

Departmental Norms: Every department has norms, and these norms can either help or hinder an instructor that wants to change the curriculum. Especially when the idea for the change comes from PER-informed results, the norms for a department could be highly traditional and that makes instigating change a larger challenge.

Expectations of Content Coverage: This refers to the often experienced problem of having too much material that needs to be covered. When there is too much material to be covered, instructors will dismiss research-based methods that are geared toward developing a deep understanding in order to get through all of the material.

Lack of Instructor Time: Lastly, instructors have a lot of responsibilities on their plate. With all that is expected of them, there is not always time to learn and integrate new techniques which is an obvious barrier to them implementing new approaches.

\section{METHODS}

Semi-structured interviews were conducted with thirty individuals, sixteen of which were in focus groups. All of the interviewees attended the PICUP FDW and were considered in some way to be a part of the PICUP community; but, the level at which they influence the community varied. The level at which they had experience teaching physics also varied with interviewees being from both universities and community colleges, and one teacher from a high school. All of these instructors were given gendered pseudonyms. These interviews were part of a larger project examining the role of the PICUP FDW in the development and expansion of a community of practice focused on integrating computation into the undergraduate curriculum. Therefore the focus of the discussion was on the PICUP community, its supports, and the individuals' roles within that community; however, a proportion of the interview was also focused on the challenges that the interviewees had faced both historically in integrating computation and the challenges they were facing at the time of the interview. The broader study is taking a phe- nomenographic approach but the analysis presented in this paper is phenomenological [10] which means that it is focused on individual experiences. Each interview was transcribed and then analyzed using a thematic analysis approach with a focus on both preconceived and emergent themes. In the first step, two researchers conducted parallel analysis and, in order to familiarize themselves with the information, read the transcripts several times. In the second step, emergent themes were developed by the researchers separately following a round of open coding where initial codes were generated. Next, initial codes were grouped into categories with each other and with preconceived codes that had also been observed in the data. The researchers compared categories and negotiated any discrepancies between interpretation of categories until a shared repertoire of categories had been developed. These categories were organized into themes which involved the combining of codes into overarching themes that accurately depict the data. The final step was for the two researchers to come to a consensus on the themes and then build a description of the theme from the categories that had informed it.

The interviews took place before, during, and after the PICUP FDW. This fact, along with the differing levels of physics that these instructors teach, and their roles in the PICUP community, make for a diverse set of experiences. Steven, for example, is a leader within PICUP and helped facilitate the FDW. He is part of the movement to change the undergraduate physics curriculum with the addition of computation, and wants to make it easy for new instructors to do this. When he started integrating computation, he was on his own entirely. His main challenge was that his department would not support him. Katelyn, on the other hand, is someone who participated in the FDW and her interview takes place after the workshop is over. She has the freedom to implement whatever she wants into her classes, but gets stuck on the little details of how to design effective activities. Her main challenge is not in dealing with her department, like it was for Steven, but knowing what to do with the support that she has. The experiences that Steven and Katelyn have are different, and so the barriers that they highlight when discussing their individual problems are different. These differences in experience between interviewees provided

\section{RESULTS}

The initial set of themes present a replication of the systemic forces that Dancy and Henderson discovered. Specifically, the PER-informed practice that is creating the barrier is the integration of computation and not PER-informed practices in general.

\section{A. Replicated results in computation context}

Student Resistance: When dealing with computation, students often resist due to the fact that they have no experi- 
ence with it. It is a daunting skill that has a reputation of being complicated, so students can decide that it is out of their wheelhouse and be resistant.

"...my experience with them [has] also been that sometimes it's almost like asking them to eat vegetables, and they don't like it, you know?" -Max

In essence, this barrier represents students rejecting learning something new that they do not think belongs in a physics class. The opinions that students have matter, and they influence how well instructors are able to integrate computation.

Time Structure: The time issue replicates almost explicitly when adding computation as a semester at an institution is, and for the most part always will be, the same fixed length.

"If I could devote the first three weeks to have the students really understand the answer to the question what is a rate...if they spent three weeks using Euler to understand what is a rate, that's three weeks well spent." -Jonah

Adding computation to a curriculum creates a new challenge of teaching a skill and a lesson that uses that skill at the same time. For example, syntax, or how to use a Jupyter Notebook, needs to be taught in addition to the physics the program is modeling. This can mean that compromises have to be made on what time is spent on, and those compromises aren't always inline with what instructors believe is valuable.

Departmental norms: This barrier is different because of the types of norms that exist when discussing computation.

"The other thing is that some of the theorists I think have expressed ...sort of second hand...some concern of courses adopting computation to the detriment of like what they feel a physics class should be, which is paper and pencil calculation." -Theo

The way that an instructor decides to start integrating computation depends on the department that they are interacting with. If the department is supportive of their efforts, it can help the process of integration happen more smoothly. But if the department has strong opinions that oppose the idea of computation, then the trouble an instructor has to go through is increased.

Expectations of Content Coverage: With computation, the amount of content that instructors need to go through can increase. This is due to the fact that an instructor cannot expect students to already know how to program, and so the basics of how a computer program works needs to be taught in addition to the physics.

"I got a lot of push back from the TAs because they were saying, 'Oh yeah, okay, this is neat, but our students still don't know how to add vectors.' Or, 'This is neat, but we have an exam coming up in two weeks and we ought to be spending this time studying what's going to be on the exam.'" -Jonah

There is a certain pressure that is put on instructors to cover the 'real physics' and have computational aspects be extra. This creates a barrier in the sense that an instructor feels their activities need to be a perfect balance of computation/physics on the first go, and that is not a reasonable expectation to have when doing something completely new.

Time of Instructors: The time of instructors is still valuable and the things that preoccupy them are still the same (e.g. large teaching loads and research).

"...[I have other goals] right now too... Chief of which is you know getting tenure. So while being an agent of change to include computation more broadly could be something that enhances my tenure profile. I'm not quite sure how I would do that right now. So I'm more focused on some of my other efforts." -Brennan

Integrating computation asks even more from instructors, especially since they may already have materials from previous semesters to use to teach a class. It can go another step further if the instructor is asked, or wants to, design an entirely new course based on computation in physics.

\section{B. New systemic forces for computation context}

Lack of Instructor Knowledge: There are plenty of instructors that want to integrate computation into their curriculum, but do not know how to start. This prevents them from being able to accomplish the goals they have for their students, and can cause other problems to appear (e.g., the department will not support them because they do not know what to do). This lack of knowledge can manifest in two different ways.

The first way it manifests is with the instructor having a lack of experience with coding themselves. This causes discomfort for an instructor as they are trying to teach something that they are not an expert on. It's difficult to write activities and educate students on a topic that is foreign to the person writing and educating.

"The only snag that I hit was when I decided to experiment with GlowScript. And, and you know, my students are... getting comfortable with vPython, then I said, you know, you could use GlowScript...but I wasn't too prepared for GlowScript myself. And so I just quickly backed off and went back to vPython." -Max

The second way is when the instructor knows how to code, and may even be a relative expert on it, but does not know how to design activities for students that are just learning. In addition to this, they may not know how to tie in valuable computational lessons to the regularly scheduled physics lessons that are found in most introductory classes. Assessing the level of student skill in computation is difficult as some students come to class with previous experience and others have never seen a piece of code before. Learning how to design activities with this in mind takes a lot of trial and error, and having someone who has done is before can be a great asset.

"I didn't need help so much with the technical language stuff, but like, you know, How should I ... You know, Should I break this up into multiple exercises? 
How long would this take? How much time should I dedicate to this? Should I have the ball without air resistance and the ball with air resistance animate at the same time, or one after the other?" -Cole

Accessible Platform: An issue that is specific to computation is that it requires a medium to be taught. This requirement hinders instructors by forcing them to evaluate what platform to use and if they can be supported using that platform.

Choosing a platform is a difficult task due to the fact that there is not a universal physics coding language that every professor uses regularly. There are pros and cons to each platform that have to be looked at, and each person has their own opinions on what the best thing to learn is. Additionally, picking a platform that is easily accessible and understandable for students is always something to be taken into consideration.

"Because our departments, some of the people said, 'Well, we know how to use MATLAB, right, use this or that.' And it wasn't until one of the astronomers spoke up and says, "Well, we're going towards using Python.' Only astronomers are. But some of the people were still saying, 'Well, why don't you keep that to a small part of your course because it's not really very important." -Jonah

The other problem is choosing a platform that is affordable and easy to access for both professors teaching a class and students alike. This is a problem that can be more common among high school teachers and community college professors, where funding is harder to come by.

"So when I first started...I wanted to have access to VPython for all the students. And what I needed was a class set of laptops. I needed VPython installed on all of them, and it took ... It actually took two years to get the funding. To get the laptops." -Chris

The IT Crowd: Nothing can be more off putting to trying something new in your classroom than having to deal with the IT department.

"One thing I'm currently doing is fighting with our IT. Obviously when you want to do something new, first taking it up with IT and saying 'Okay, I have no expe- rience how to do it. Can you help me?' That usually doesn't go very far, so that's why I implemented the original JupyterHub server on my own office work station. But we have a new work station policy...they want everything to be secure, secure, secure...they closed the ports on which I run my JupyterHub server." -Dan

The issue that Dan is highlighting is one that is unique to integrating computation. It is unique because computation requires the use of computers, which can be under the control of a department if students don't have their own, and an instructor needs a way to both give and receive assignments, which has many effective methods. Ultimately, if an instructor wants to use resources that are already available via the institution, there is a good chance they'll have to go through their IT department.

\section{v. DISCUSSION AND CONCLUSION}

The results of the interviews in the previous sections demonstrate that there are both barriers specific to computation and barriers that have been identified previously in Henderson and Dancy's research that occur when integrating computation into the undergraduate curriculum. This work, in part, acted as a replication study of Henderson and Dancy's work and reinforces that these barriers to implementing changes to curricula are present despite the differences in the type of implementation being attempted. It also demonstrates that although the barriers may reoccur in the computational context, there are definitive contextual differences in the barriers.

The first step in helping to solve any problem is identifying it. By identifying the barriers mentioned above to integrating computation into the undergraduate curriculum, we have provided a foundation for future work that will focus on understanding how to support faculty from facing these barriers or navigating them when they are encountered. It also provides research-based discussion points for the PICUP workshop. PICUP is a resource for those who are at any level of integrating computation. The more we know and learn about the issues surrounding integrating computation, the easier it will be for new instructors to join the community of those teaching computation to their students.
[1] N. Chonacky and D. Winch, American Journal of Physics (2008), 10.1119/1.2837811.

[2] M. D. Caballero, ArXiv 1712.07701 , 1 (2017).

[3] NGSS Lead States, Achieve, Inc. on behalf of the twenty-six states and partners that collaborated on the NGSS , 1 (2013).

[4] M. M. Cooper, M. D. Caballero, D. Ebert-May, C. L. FataHartley, S. E. Jardeleza, J. S. Krajcik, J. T. Laverty, R. L. Matz, L. A. Posey, and S. M. Underwood, Science 350, 281 (2015).

[5] AAPT Undergraduate Curriculum Task Force, American As- sociation of Physics Teachers (2016).

[6] P. W. Irving, M. J. Obsniuk, and M. D. Caballero, European Journal of Physics 38, 0 (2017).

[7] P. W. Irving and M. D. Caballero, , 188 (2017).

[8] C. Henderson and M. H. Dancy, AIP Conference Proceedings 790, 109 (2005).

[9] M. H. Dancy and C. Henderson, AIP Conference Proceedings 790, 113 (2005).

[10] C. Moustakas, Phenomenological research methods (Sage, 1994) 